\title{
Non-Celiac Gluten Sensitivity: A Challenging Diagnosis in Children with Abdominal Pain
}

\author{
Frank M. Ruemmele \\ Université Paris Descartes, Sorbonne Paris Cité, Paris, France; Assistance Publique-Hôpitaux de Paris, Hôpital \\ Necker-Enfants Malades, Service de Gastroentérologie Pédiatrique, Paris, France; Institute IMAGINE INSERM U1163, \\ Paris, France
}

\section{Key Messages}

- Gluten ingestion can cause different overlapping clinical disorders: three distinct entities are recognized: celiac disease, wheat allergy, and non-celiac gluten sensitivity.

- The incidence of non-celiac gluten sensitivity is steadily increasing in adults as well as in children and adolescents.

- Non-celiac gluten sensitivity is a syndrome of intestinal and extraintestinal manifestations in response to the ingestion of gluten; however, newer data indicate that other components of wheat, such as amylase/trypsin inhibitors, wheat germ agglutinins, or FODMAPs, may also cause clinical symptoms. FODMAPs are present in many nutrients other than cereals.

\section{Keywords}

Gluten · FODMAPs · Irritable bowel syndrome · Gluten sensitivity $\cdot$ Pediatrics

\footnotetext{
Abstract

Several disorders related to the ingestion of gluten are well recognized despite overlapping clinical presentations: celiac
}

disease, an autoimmune enteropathy triggered by gluten ingestions in susceptible individuals, allergy to wheat, and more recently non-celiac gluten sensitivity (NCGS). While celiac disease and wheat allergy are well-known disorders with a clear-cut diagnosis based on clinical tests and biological parameters, NCGS is a more difficult diagnosis, especially in children with functional gastrointestinal (Gl) complaints. NCGS is considered a syndrome of intestinal but also extraintestinal symptoms occurring within hours, but sometimes even after several days of gluten ingestion. In children, the leading symptoms of NCGS are abdominal pain and diarrhea, while extraintestinal symptoms are rare, in contrast to adult patients. No precise diagnostic test nor specific biomarkers exist, except a rather cumbersome three-phase gluten-exposure, gluten-free diet, followed by a blinded placebo-controlled gluten challenge with crossover to provoke symptoms elicited by gluten in a reproducible manner that disappear on gluten-free alimentation. Recent data indicate that the peptide part of wheat proteins is not necessarily the sole trigger of clinical symptoms. Mono- or oligosaccharides, such as fructan and other constituents of wheat, were able to provoke Gl symptoms in clinical trials. These new findings indicate that the term gluten sensitivity is probably too restrictive. The incidence of NCGS was reported in the range of $1-10 \%$ in the general population and to increase steadily; however, most data are based on patients' self-reported gluten intolerance or avoidance without a medically confirmed

\section{KARGER}

(C) 2019 S. Karger AG, Basel

E-Mail karger@karger.com

www.karger.com/anm
Professor Frank M. Ruemmele, MD, PhD

Pediatric Gastroenterology, Hôpital Necker-Enfants Malades

149 rue de Sèvres

FR-75015 Paris (France)

E-Mail frank.ruemmele@nck.aphp.fr 
diagnosis. Treatment consists of gluten avoidance for at least several weeks or months. Patients with NCGS require regular reassessment for gluten tolerance allowing with time the reintroduction of increasing amounts of gluten.

(C) 2019 S. Karger AG, Basel

\section{Introduction}

Gastrointestinal (GI) disorders related to gluten or wheat ingestion are becoming increasingly diagnosed. At least three distinct disorders related to gluten exposure are recognized: celiac disease, wheat allergy, and non-celiac gluten sensitivity (NCGS). Diagnostic criteria for celiac disease and wheat allergy are very stringent and several specific disease markers exist, while NCGS lacks a specific biological diagnostic marker. Celiac disease is a life-long autoimmune disease that occurs in genetically susceptible individuals (HLA DQ2 or DQ8) upon the ingestion of gliadin. Pediatricians are well aware of the clinical symptoms (diarrhea, abdominal distension, weight loss and growth failure, as well as extraintestinal signs such as anemia, fatigue, mood alteration, skin involvement, etc.). The diagnostic screen is based on the presence of autoantibodies (anti-endomysium and antitransglutaminase IgA antibodies) and confirmed by the presence of duodenal villous atrophy with an increased number of intraepithelial lymphocytes [1]. In children with a classical presentation and at least 10 -fold elevated autoantibodies, HLA compatible with celiac disease, a duodenal biopsy is no more indispensable before starting a gluten-free diet (GFD) [2]. Similarly, the diagnosis of wheat allergy is relatively clear-cut, and pediatricians, allergologists, and pediatric gastroenterologists easily evoke this diagnosis in a child presenting with allergic GI, skin, or respiratory symptoms usually minutes after the ingestion of wheat or gluten. Specific IgE in conjunction with skin prick and patch tests allow further evoking this diagnosis prior to starting an exclusion diet $[3,4]$.

In contrast to celiac disease and wheat allergy, NCGS is relatively unknown to pediatricians and pediatric specialists. As for celiac disease and wheat allergy, in patients with NCGS, symptoms appear after the ingestion of gluten after a variable interval of few hours up to sometimes several days. Since diagnostic biomarkers are missing, the diagnosis of NCGS can be only suspected once celiac disease and wheat allergy have been formally excluded, since clinical presentations largely overlap. The only reliable diagnostic test that confirms the suspected diagnosis is a double-blind gluten/placebo challenge [5].

The first description of NGCS goes back to the late seventies reporting 8 women with abdominal pain and diarrhea related to the ingestion of gluten, disappearing on GFD, but reappearing upon a gluten challenge [6]. However, only about 30 years later, this entity was fully recognized by gastroenterologists and first reports in children are quite recent and date from 2014 [7]. To improve the diagnostic criteria and to better characterize NCGS, an expert meeting, published as the Salerno Experts' criteria, took place in 2014, defining NCGS as a "syndrome of intestinal and extra-intestinal symptoms related to the ingestion of gluten-containing foods" [8].

\section{Epidemiological Studies on NCGS}

Over the last couple of years, GFD became very fashionable and it is estimated that up to $20 \%$ of Americans choose to follow a GFD, most often without a medical advice. Thus, GFD is on an exponential rise - due to this new demand, a quickly growing industry offering an increasing panel of gluten-free products parallels this phenomenon. Most often this gluten avoidance is motivated by the observation of "feeling better" on GFD without a medical diagnosis of a gluten-related disorder. Since specific diagnostic markers are missing, the large majority of data on NCGS are based on patients' self-reported gluteninduced symptoms. Several studies tried to estimate the prevalence of NCGS: one of the first reports comes from the Center for Celiac Research at the University of Maryland (USA) reporting a prevalence of gluten avoidance of approximately $6 \%$ in a population of patients with GI symptoms seen at a tertiary center [9], while other reports indicate a prevalence of 0.5 up to $10 \%$ in a general population [10-13]. All studies show a clear predominance of women. The huge variability of prevalence estimates reflects the fact that these data are based on patient-reported symptoms in relation to gluten consumption excluding patients diagnosed with celiac disease or wheat allergy. Few reports indicate that NCGS is not restricted to adults, but it may also occur in children and adolescents. A recent Sicilian study in 555 high school students (mean age 17 years) indicated a prevalence of self-reported NCGS of $12.2 \%$ with the leading symptom irritable bowel syndrome (IBS) [14]. However, less than
Ruemmele 


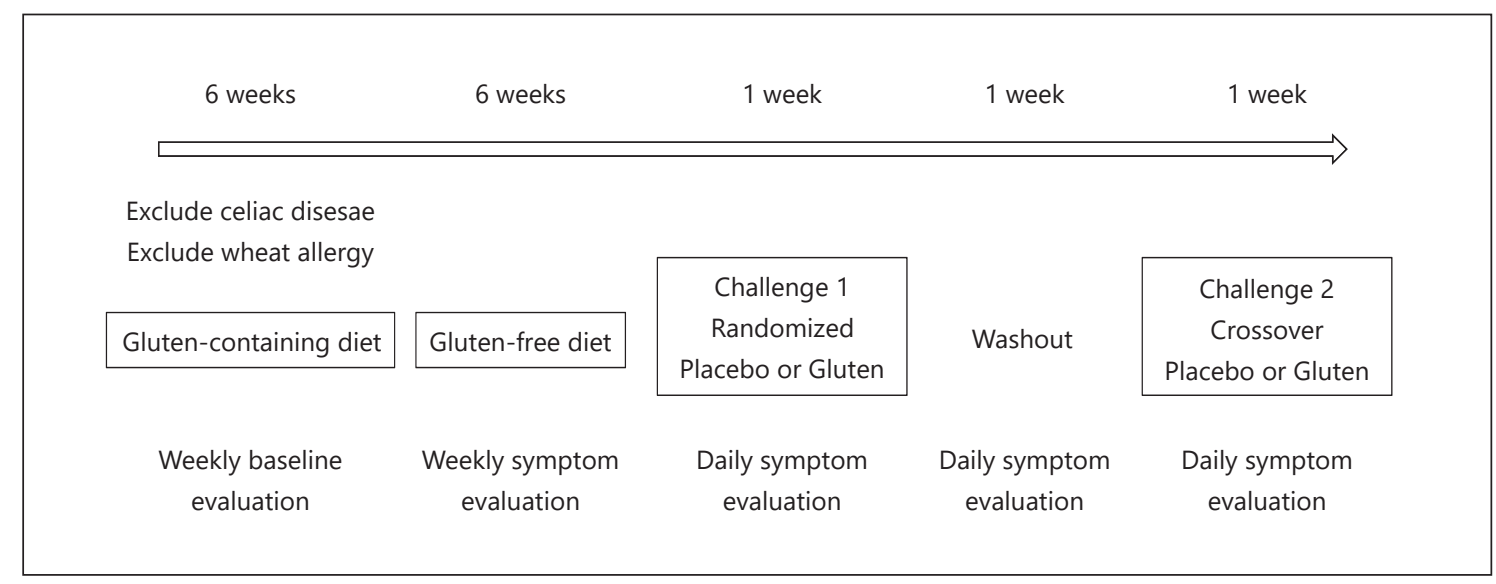

Fig. 1. Suggested algorithm for the diagnosis of non-celiac gluten sensitivity (Salerno Experts' criteria). While on a gluten-containing diet, exclude celiac disease and wheat allergy. Evaluate baseline symptoms (questionnaire) and quantitate one of the three most significant symptoms on a weekly basis. After at least 6 weeks of a gluten-containing diet, switch to a 6-week strict gluten-free diet with weekly evaluation of symptoms. If there is a decrease of symp- toms of $30 \%$ or more, perform a double-blind crossover challenge with either the sequence "placebo - washout - gluten challenge" or the sequence "gluten challenge - washout - placebo." During these weekly challenges or washout, perform daily symptom evaluation allowing a firm diagnosis of appearance of symptoms caused by gluten ingestion. a quarter was consulting for medical advice. A markedly lower but still impressive rate (5.2\%) of gluten avoidance in children (median age 10.6 years) was reported in a subanalysis of the New Zealand Asthma and Allergy Cohort Study [15]. It is interesting to note that studies based on a double-blind placebo-controlled approach to diagnose NCGS (see below) indicate that more than two-thirds of patients experiencing gluten sensitivity do not react in a reproducible manner to the gluten challenge suggesting that the prevalence of NCGS based on a clear-cut diagnosis is reduced to at least half if not less compared to the numbers reported so far. In comparison, even after correcting the estimates, NCGS seems to be two or three times more prevalent than celiac disease (with an estimated prevalence for celiac disease of $1 \%$ ).

\section{Clinical Presentation and Diagnosis of NCGS}

The clinical presentation of NCGS is variable with multiple intestinal and extraintestinal symptoms occurring within hours, but in some patients only after several days upon the ingestion of gluten. The Salerno Experts' criteria [8] list bloating and abdominal pain as the most frequent GI symptoms, followed by diarrhea, epigastric pain, nausea, and alternating bowel habits or constipation. Some patients also report oral aphthous ulcerations or reflux symptoms evoking gastroesophageal disease. Frequent extraintestinal symptoms are a general lack of wellbeing, headache, tiredness, anxiety, but also muscle and joint pain, skin rash/dermatitis, anemia, numbness or foggy mind are reported. Even less specific symptoms such as sleeping disturbances, mood swings, or hallucinations were reported after gluten ingestion. The great majority of intestinal symptoms overlap with classical celiac disease and even more importantly with either forms of IBS, diarrheal IBS, or IBS with constipation [16]. A small pediatric study on 15 children with gluten sensitivity reported that intestinal symptoms are clearly predominant with abdominal pain as the lead symptom $(80 \%)$, followed by diarrhea $(73 \%)$, while extraintestinal symptoms were markedly less frequent [7].

Given the high variability of clinical signs, the precise cause relationship (gluten ingestion provoking clinical symptoms) has to be established in a very distinct manner. Unfortunately, no specific biomarker for NCGS exists. Many markers were tested in the past and there are some reports indicating that anti-gliadin (AGA) IgG antibodies are positive in over $50 \%$ of patients with suspected NCGS [17] and AGA IgG tend to become negative under GFD in patients with NCGS [18]. However, it was pointed out that AGA probably develop in a majority of patients with an altered (increased) gut permeability without any disease specificity, thus this finding has to be considered with caution [19]. Other markers tested are in vitro basophil activation test in response to food antigens [20] or the number of eosinophils in the intestinal mu- 
Table 1. Symptom questionnaire for the diagnosis of NCGS [22]

- Abdominal pain or discomfort
- Heartburn
- Acid regurgitation
- Bloating
- Nausea and vomiting
- Borborygmus
- Abdominal distension
- Eructation
- Increased flatus
- Decreased passage of stools
- Increased passage of stools
- Loose stools
- Hard stools
- Urgent need for defecation
- Feeling of incomplete evacuation
- Extraintestinal symptoms
- Dermatitis
- Headache
- Foggy mind
- Fatigue
- Numbness of the limbs
- Joint/muscle pains
- Fainting
- Oral/tongue lesions
- Other (specify)

cosa [21]. However, neither test reached a high level of specificity, making them not usable in a routine clinical setting.

The gold standard and so far the sole diagnostic test allowing the confirmation of true gluten sensitivity causing intestinal and/or extraintestinal symptoms upon gluten ingestion is a double-blind placebo-controlled gluten challenge with crossover, as highlighted in the Salerno criteria [8]. The experts recommend a full diagnostic procedure (Fig. 1): patients on a gluten-containing diet experiencing symptoms after the consumption of gluten are tested for celiac disease or allergic reactions to gluten or wheat. Once celiac disease and wheat allergy are ruled out, patients will be put on a GFD for at least 6 weeks. Most often symptoms disappear very quickly within few days, especially GI symptoms. However, a prolonged GFD is necessary since particularly unspecific symptoms such as fatigue, mood changes, or headache may persist for several weeks after the ingestion of gluten. One challenge consists in how to evaluate the decrease/disappearance of symptoms on GFD and reappearance on gluten challenge: the Salerno criteria suggest the use of a modified Gastrointestinal Symptom Rating Scale (GSRS) [22] (Table 1); while on a normal gluten-containing diet each symptom should be evaluated on a weekly basis starting at week 2 prior to the introduction of a GFD. This baseline evaluation serves as comparator to the weekly evaluation on GFD. To measure on a quantitative level, one of the three main clinical symptoms is assessed numerically using a Numerical Rating Scale (NRS) with a score ranging from 1 (mild) to 10 (severe) [8]. A drop of at least $30 \%$ on the NRS for at least $50 \%$ of the time is considered as a symptomatic response indicating a likely link between the ingestion of gluten and experienced clinical symptoms. However, the ultimate confirmation of the diagnosis of NCGS requires a double-blind placebo-controlled challenge with crossover. The gluten challenge should be based on a daily intake of at least $8 \mathrm{~g}$ of gluten with a defined amylase/trypsin inhibitor (ATI) content (see below) and free of fermentable oligo-, di-, and monosaccharides and polyols (FODMAPs) over 1 week (in patient with fluctuating symptoms even longer), followed by 1 week washout and a crossover to placebo for another week. It must be assured that the vehicle for the placebo is truly gluten-free. Ideally, patients and physicians are both blinded to the protocol allowing unbiased evaluation. Symptoms are evaluated on a daily basis as above and a variation of at least $30 \%$ on gluten challenge versus placebo is considered significant. The arbitrary $30 \%$ cutoff has to be validated in the future by independent studies. In daily clinical practice, this diagnostic setting is often not possible, since patients are already on a gluten avoidance diet and not willing to increase their gluten intake for a prolonged period to allow a proper baseline evaluation. Thus, a diagnostic challenge is a pragmatic way to evoke the diagnosis of NCGS. In addition, in routine clinical care a double-blind approach is not very realistic; therefore, most settings propose a single-blinded testing that sometimes needs to be repeated, since many patients are prone to experience strong nocebo effects, complicating the interpretation of the results.

Patients that test negative on a blinded gluten challenge have to be examined for other causes of IBS-like symptoms, especially FODMAP intolerance. The recent report of Skodje et al. [23] clearly highlights the importance of FODMAPs in patients' symptoms. They performed a double-blind crossover challenge in 59 adults with self-reported gluten sensitivity (celiac disease was formally excluded) with a random assignment to receive muesli bars containing placebo, fructan $(2.1 \mathrm{~g})$, or gluten (5.7 g) for 7 days. Participants crossed over to each study arm after a minimum 7 -day washout period allowing that all 59 individuals completed the three challenges. Based on the GSRS-IBS, 24 participants showed highest scores 
while consuming fructan, 22 on placebo, and only 13 on gluten. This indicates that fructan might be a major trigger for clinical symptoms in patients reporting gluten intolerance. This Norwegian study further extended the findings from a previous study from Peter Gibson's group in Australia [24]: in 37 individuals with self-reported NGCS put on a FODMAP reduction diet prior to a challenge with high gluten (16 g), low gluten ( $2 \mathrm{~g})$, or placebo, already the FODMAP restriction significantly reduced clinical symptoms. On challenge, all patients reported symptoms that increased with the duration of the challenge independent of the treatment arm: $30 \%$ of participants showed symptoms in response to placebo and overall gluten-specific effects were only observed in $8 \%$. The observation of a high nocebo rate complicates the interpretation of these challenges. The recent report on 28 children with presumed NCGS further underlies the difficulty of diagnosis [25]. This first randomized doubleblind placebo-controlled crossover trial in children was based on three steps: initially 1,114 children with chronic functional GI symptoms alone or in combination with extraintestinal symptoms were screened for a potential link of symptoms to gluten ingestion. $96.7 \%$ did not show any relation of symptoms to gluten-containing food. Finally, only 36 children were included in the study with 5 children improving during the run-in phase (2-week exposure to a gluten-containing diet for baseline evaluation). 31 children had an open GFD over 2 weeks, 3 did not respond, thus only 28 children (mean age 11.4 years) entered into the placebo-controlled crossover trial with a 2 -week gluten/placebo challenge, 1-week washout, and another 2-week crossover. Eleven children (39\%) showed a gluten-specific response based on a numerical score of the perception of GI symptoms; however, no difference was observed in the severity of the global score comparing challenges with gluten to placebo, in addition no differences were observed between the placebo and glutenchallenged group when analyzing any biological parameters included in this study.

\section{Pathophysiology of NCGS}

The diagnostic difficulties and uncertainties, even in specific clinical trials, reflect a potentially more complex pathophysiology of NCGS than previously suspected (Fig. 2). The current notion that clinical symptoms are triggered in response to the ingestion of gluten proteins seems too simplistic. Gluten is a storage protein of wheat, rye, and barley and it accounts approximately for $75 \%$ of the total grain protein of the endosperm, the remaining

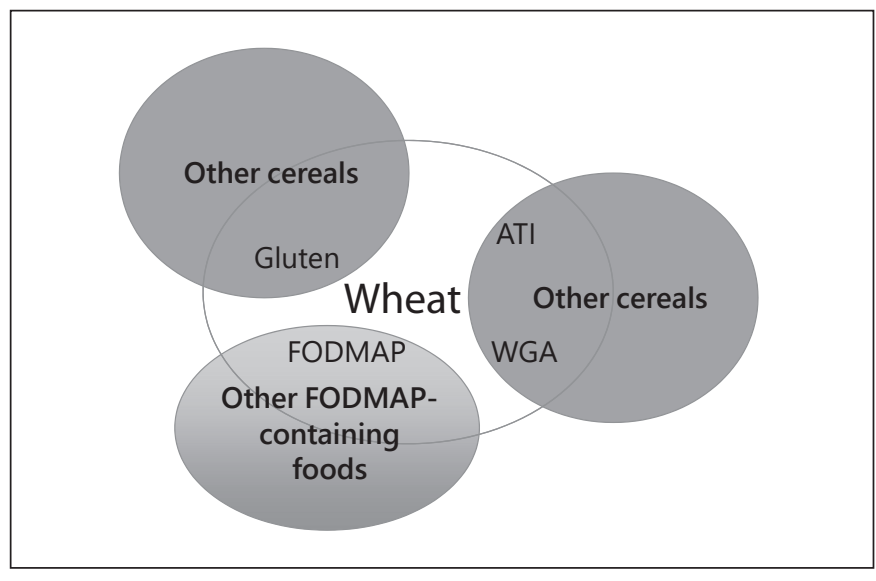

Fig. 2. Non-celiac gluten sensitivity is a syndrome of intestinal and extraintestinal manifestations after the ingestion of gluten. However, the pathophysiology is more complex, since patients report symptoms after the ingestion of wheat, but these are only partially relieved on a gluten-free diet. This might reflect the fact that other components of wheat or other cereals, such as wheat germ agglutinins (WGA), amylase/trypsin inhibitor (ATI), or fermentable oligo-, di-, and monosaccharides and polyols (FODMAPs) might trigger the symptoms. Since some components are shared with other nutrients, such as fruits or vegetables (for FODMAP), a more in-depth analysis is necessary for patients still symptomatic on a strict gluten-free diet.

proteins being globulin and albumin. Gluten can be separated into monomeric (gliadins) and polymeric prolamines (glutenines). In patients with celiac disease, several gliadin-derived oligopeptides were identified to elicit a specific humoral immune response, while a $\mathrm{N}$ terminal peptide from alpha-gliadin is supposed to induce innate immune responses in celiac disease patients $[1,26]$. So far, no specific gluten peptide was identified to be reproducibly associated with NCGS or to trigger specific symptoms. It is important to underline that wheat contains many other components that might be responsible for the symptoms of patients: ATI, lipopolysaccharides, wheat germ agglutinins (WGA), and FODMAPs.

ATI are protective proteins in several cereals including wheat, rye, and barley. They are part of a plant's defensive system and protect against parasites by inhibiting their amylase or trypsin-like enzymes. ATI are highly packed together with gluten in the endosperm, and ATI content differs markedly between modern bread wheat (very high content) compared to older and less complex wheats (low content). ATI are the major allergen causing baker's asthma [27] and it is well known that ATI trigger innate immune responses via the direct stimulation of Toll-like re- 
ceptor 4, thereby enhancing an inflammatory response. When performing a diagnostic challenge for NCGS, it must be assured that the gluten vehicle also contains ATI at the suggested ratio of $0.3 \mathrm{~g} \mathrm{ATI} / 8 \mathrm{~g}$ of gluten [8].

WGA are also protective proteins binding to virtually all cell types including other plants and fungi; they are highly packed in the germ of wheat grains. As for ATI, WGA are very resistant to heat and proteolysis. Such as lectins, WGA are considered as anti-nutrients within food. Wheat germs contain the highest WGA concentrations (up to $0.5 \mathrm{~g} / \mathrm{kg}$ [28]) and in its unprocessed form (i.e., in muesli), a markedly higher WGA activity was found compared to foods containing processed wheat germs. WGA have a high potential to bind to human $\mathrm{N}$ acetlyneuraminic acid expressed on cell surfaces, particularly on the glykocalix of enterocytes and immune cells. Upon binding, WGA induce strong proinflammatory responses [29]. Murine perito-

neal macrophages responded with high proinflammatory cytokine secretion to WGA stimulation in vitro (TNF- $\alpha$, IL-1 $\beta$, IL-12, and IFN- $\gamma$ ) [30]. Similarly, stimulation of isolated human PBMC with minimal amounts of WGA elicited the release of proinflammatory cytokines [31]. These results indicate that WGA have a high potential to initiate or maintain inflammatory responses. In the intestinal tract, this can lead to a disruption of the intestinal epithelial barrier increasing permeability.

Human in vivo data confirming these inflammatory reactions to WGA ingestion are still lacking; however, antibodies to WGA have been detected in the serum of healthy volunteers [32] and in patients with celiac disease high levels of anti-WGA antibodies were measured.

FODMAPs comprise mono-, di-, and oligosaccharides of fructose (fructans) and galacto-oligosaccharides (GOS) as well as polyols, such as sorbitol, mannitol, and xylitol that are partially, poorly, or not at all digested and thus fermented by the microbiome in the colon leading to gas production and colonic distension responsible for discomfort and pain. FODMAPs play an important role in the pathogenesis of IBS and, as already discussed, apparently also for patients with gluten sensitivity. This is not surprising, since wheat contains variable amounts of fructans depending on the fermentation process. An Australian study analyzed 55 commonly consumed breakfast cereals, breads, pulses, grains, and biscuits indicating significant fructan concentrations even in gluten-free prod- ucts [33]. Spelt bread had the lowest fructan content in this analysis, helping to understand why patients with IBS tolerate spelt bread better than others.

\section{Treatment Options of NCGS}

Once the link between gluten ingestion and clinical symptoms is confirmed, a strict GFD is indicated for several weeks to months. However, wheat contains several other elements (ATI, WGA, FODMAPs) that can trigger symptoms potentially confusing the clinical situation. The reduction to gluten as sole causative food ingredient is too simplistic. As discussed, nutrients other than gluten can maintain the clinical complaints in patients. The best example is fructan, present in wheat, but also in many fruits and vegetables. Therefore, a precise exploration with repeat challenges might help to further improve the clinical situation of a patient feeling some relieve but not completely symptom-free on GFD. There are no clear recommendations for the duration of a GFD, but it is suggested to last for several weeks to months and thereafter reassess gluten tolerance allowing to re-introduce small amounts of gluten. It is important to mention that a GFD alters the microbial homeostasis and a reduction of lactobacilli and bifidobacteria in the gut were observed. Calcium, iron, and folate status have to be monitored on GFD, since some reports indicate a reduced intake on GFD leading potentially to deficiency.

It is important to note that the perception of glutenfree food equals healthier food is erroneous. A recent study by Elliott [34] revealed that gluten-free products had a lower sodium level and reduced total fat, but also less protein and a high percentage of sugar-derived calories, comparable to food without a gluten-free claim. Both types of products were considered as of poor nutritional value.

\section{Future Perspectives}

There is a major need to improve the diagnostic arsenal for NCGS and if possible to develop clear biomarkers avoiding the quite cumbersome and time-consuming challenge tests. The notion of gluten as the sole trigger of NCGS is too restrictive; recent clinical trials indicate that other food components, such as FODMAPs also play an important role. Thus, future clinical research has to ad-
Ruemmele 
dress this point and determine more precisely the role of different foods and nutrients. Since many nutrients contain similar ingredients, the spectrum of different foods associated with NCGS might increase markedly. This is important, since many patients suffer for a long time, while a GFD completely changes their lives; this is not the case for all patients who eventually have other needs. On the other hand, gluten avoidance is becoming more and more fashionable and reflects a certain "healthy" lifestyle, without necessarily indicating a medical need. This trend should be seen with caution particularly for children, since gluten-free products are not necessarily of good nutritional quality, they are quite expensive, and a strict and prolonged GFD might lead to deficiencies. Therefore, clear information for the general population is important to avoid any abuse of restriction diets particularly for children.

\section{Disclosure Statement}

F.M.R. has received speaker fees from Schering Plough, Nestlé, Mead Johnson, Ferring, MSD, Janssen, Centocor, Abbvie, serves as a board member for SAC: DEVELOP (Janssen), CAPE (AbbVie), LEA (Abbvie), and has been invited to MSD France, Nestlé Nutrition Institute, Nestlé Health Science, AbbVie, Danone, Mead Johnson, TAKEDA, BIOGEN, PFIZER, ARKOPHARMA, SHIRE.

The writing of this article was supported by Nestle Nutrition Institute.

\section{References}

1 Garnier-Lengliné $H$, Cerf-Bensussan N, Ruemmele FM. Celiac disease in children. Clin Res Hepatol Gastroenterol. 2015 Oct; 39(5):544-51.

2 Husby S, Koletzko S, Korponay-Szabó IR, Mearin ML, Phillips A, Shamir R, et al.; ESPGHAN Working Group on Coeliac Disease Diagnosis; ESPGHAN Gastroenterology Committee; European Society for Pediatric Gastroenterology, Hepatology, and Nutrition. European Society for Pediatric Gastroenterology, Hepatology, and Nutrition guidelines for the diagnosis of coeliac disease. J Pediatr Gastroenterol Nutr. 2012 Jan;54(1):136-60.

3 Soares-Weiser K, Takwoingi Y, Panesar SS, Muraro A, Werfel T, Hoffmann-Sommergruber K, et al.; EAACI Food Allergy and Anaphylaxis Guidelines Group. The diagnosis of food allergy: a systematic review and metaanalysis. Allergy. 2014 Jan;69(1):76-86.

4 Bischoff S, Crowe SE. Gastrointestinal food allergy: new insights into pathophysiology and clinical perspectives. Gastroenterology. 2005 Apr;128(4):1089-113.

5 Catassi C, Alaedini A, Bojarski C, Bonaz B, Bouma G, Carroccio A, et al. The Overlapping Area of Non-Celiac Gluten Sensitivity (NCGS) and Wheat-Sensitive Irritable Bowel Syndrome (IBS): an Update. Nutrients. 2017 Nov;9(11): 1268.

6 Ellis A, Linaker BD. Non-coeliac gluten sensitivity? Lancet. 1978 Jun;1(8078):1358-9.

7 Francavilla R, Cristofori F, Castellaneta S, Polloni C, Albano V, Dellatte S, et al. Clinical, serologic, and histologic features of gluten sensitivity in children. J Pediatr. 2014 Mar; 164(3):463-7.e1.

8 Catassi C, Elli L, Bonaz B, Bouma G, Carroccio A, Castillejo G, et al. Diagnosis of NonCeliac Gluten Sensitivity (NCGS): The Salerno Experts' Criteria. Nutrients. 2015 Jun;7(6): 4966-77.
9 Sapone A, Bai JC, Ciacci C, Dolinsek J, Green $\mathrm{PH}$, Hadjivassiliou M, et al. Spectrum of gluten-related disorders: consensus on new nomenclature and classification. BMC Med. 2012 Feb;10(1):13.

10 DiGiacomo DV, Tennyson CA, Green PH, Demmer RT. Prevalence of gluten-free diet adherence among individuals without celiac disease in the USA: results from the Continuous National Health and Nutrition Examination Survey 2009-2010. Scand J Gastroenterol. 2013 Aug;48(8):921-5.

11 Volta U, Bardella MT, Calabrò A, Troncone R, Corazza GR; Study Group for Non-Celiac Gluten Sensitivity. An Italian prospective multicenter survey on patients suspected of having non-celiac gluten sensitivity. BMC Med. 2014 May;12(1):85.

12 Ontiveros N, López-Gallardo JA, Vergara-Jiménez MJ, Cabrera-Chávez F. Self-Reported Prevalence of Symptomatic Adverse Reactions to Gluten and Adherence to GlutenFree Diet in an Adult Mexican Population. Nutrients. 2015 Jul;7(7):6000-15.

13 van Gils T, Nijeboer P, IJssennagger CE, Sanders DS, Mulder CJ, Bouma G. Prevalence and Characterization of Self-Reported Gluten Sensitivity in The Netherlands. Nutrients. 2016 Nov;8(11):714

14 Carroccio A, Giambalvo O, Blasca F, Iacobucci R, D’Alcamo A, Mansueto P. Self-Reported Non-Celiac Wheat Sensitivity in High School Students: Demographic and Clinical Characteristics. Nutrients. 2017 Jul;9(7):771.

15 Tanpowpong P, Ingham TR, Lampshire PK, Kirchberg FF, Epton MJ, Crane J, et al.; New Zealand Asthma and Allergy Cohort Study Group. Coeliac disease and gluten avoidance in New Zealand children. Arch Dis Child. 2012 Jan;97(1):12-6.
16 Drossman DA. Functional Gastrointestinal Disorders: History, Pathophysiology, Clinical Features and Rome IV. Gastroenterology. 2016 Feb;150(6):S0016-5085(16)00223-7.

17 Volta U, Tovoli F, Cicola R, Parisi C, Fabbri A, Piscaglia M, et al. Serological tests in gluten sensitivity (nonceliac gluten intolerance). J Clin Gastroenterol. 2012 Sep;46(8):680-5.

18 Caio G, Volta U, Tovoli F, De Giorgio R. Effect of gluten free diet on immune response to gliadin in patients with non-celiac gluten sensitivity. BMC Gastroenterol. 2014 Feb;14(1): 26.

19 Feldman MF, Bird JA. Clinical, serologic, and histologic features of gluten sensitivity in children. Pediatrics. 2014 Nov;134 Suppl 3: S157-8.

20 Carroccio A, Brusca I, Mansueto P, Pirrone G, Barrale M, Di Prima L, et al. A cytologic assay for diagnosis of food hypersensitivity in patients with irritable bowel syndrome. Clin Gastroenterol Hepatol. 2010 Mar;8(3):25460.

21 Carroccio A, Giannone G, Mansueto P, Soresi M, La Blasca F, Fayer F, et al. Duodenal and Rectal Mucosa Inflammation in Patients With Non-celiac Wheat Sensitivity. Clin Gastroenterol Hepatol. 2018 Aug; S1542-3565(18)30881-4.

22 Kulich KR, Madisch A, Pacini F, Piqué JM, Regula J, Van Rensburg CJ, et al. Reliability and validity of the Gastrointestinal Symptom Rating Scale (GSRS) and Quality of Life in Reflux and Dyspepsia (QOLRAD) questionnaire in dyspepsia: a six-country study. Health Qual Life Outcomes. 2008 Jan;6(1):12.

23 Skodje GI, Sarna VK, Minelle IH, et al. Fructan, Rather Than Gluten, Induces Symptoms in Patients With Self-Reported Non-Celiac Gluten Sensitivity. Gastroenterology. 2018; 154:529-39.e2 
24 Biesiekierski JR, Peters SL, Newnham ED, et al. No effects of gluten in patients with selfreported non-celiac gluten sensitivity after dietary reduction of fermentable, poorly absorbed, short-chain carbohydrates. Gastroenterology. 2013;145:320-8.e1-3.

25 Francavilla R, Cristofori F, Verzillo L, Gentile A, Castellaneta S, Polloni C, et al. Randomized Double-Blind Placebo-Controlled Crossover Trial for the Diagnosis of Non-Celiac Gluten Sensitivity in Children. Am J Gastroenterol. 2018 Mar;113(3):421-30.

26 Meresse B, Malamut G, Cerf-Bensussan N. Celiac disease: an immunological jigsaw. Immunity. 2012 Jun;36(6):907-19.

27 Sander I, Rihs HP, Doekes G, Quirce S, Krop E, Rozynek P, et al. Component-resolved diagnosis of baker's allergy based on specific IgE to recombinant wheat flour proteins. J Allergy Clin Immunol. 2015 Jun;135(6):1529-37.
28 de Punder K, Pruimboom L. The dietary intake of wheat and other cereal grains and their role in inflammation. Nutrients. 2013 Mar; 5(3):771-87.

29 Hurley BP, Pirzai W, Eaton AD, Harper M, Roper J, Zimmermann C, et al. An experimental platform using human intestinal epithelial cell lines to differentiate between hazardous and non-hazardous proteins. Food Chem Toxicol. 2016 Jun;92:75-87.

30 Sodhi A, Kesherwani V. Production of TNFalpha, IL-1beta, IL-12 and IFN-gamma in murine peritoneal macrophages on treatment with wheat germ agglutinin in vitro: involvement of tyrosine kinase pathways. Glycoconj J. 2007 Dec;24(9):573-82.
31 Dalla Pellegrina C, Perbellini O, Scupoli MT, Tomelleri C, Zanetti C, Zoccatelli G, et al. Effects of wheat germ agglutinin on human gastrointestinal epithelium: insights from an experimental model of immune/epithelial cell interaction. Toxicol Appl Pharmacol. 2009 Jun;237(2):146-53.

32 Tchernychev B, Wilchek M. Natural human antibodies to dietary lectins. FEBS Lett. 1996 Nov;397(2-3):139-42.

33 Biesiekierski JR, Rosella O, Rose R, Liels K, Barrett JS, Shepherd SJ, et al. Quantification of fructans, galacto-oligosacharides and other short-chain carbohydrates in processed grains and cereals. J Hum Nutr Diet. 2011 Apr;24(2):154-76.

34 Elliott C. The Nutritional Quality of GlutenFree Products for Children. Pediatrics. 2018 Aug;142(2):142. 